\title{
Improper ferroelectric polarization in a perovskite driven by intersite charge transfer and ordering
}

\author{
Wei-Tin Chen,,${ }^{1,2}$ Chin-Wei Wang, ${ }^{3}$ Hung-Cheng Wu, ${ }^{4}$ Fang-Cheng Chou, ${ }^{1,2,3}$ Hung-Duen Yang, ${ }^{4}$ Arkadiy Simonov, ${ }^{5}$ \\ and M. S. Senn ${ }^{6, *}$ \\ ${ }^{1}$ Center for Condensed Matter Sciences, National Taiwan University, Taipei 10617, Taiwan \\ ${ }^{2}$ Taiwan Consortium of Emergent Crystalline Materials, Ministry of Science and Technology, Taipei 10622, Taiwan \\ ${ }^{3}$ National Synchrotron Radiation Research Center, Hsinchu 30076, Taiwan \\ ${ }^{4}$ Department of Physics, National Sun Yat-Sen University, Kaohsiung 80424, Taiwan \\ ${ }^{5}$ Department of Chemistry, University of Oxford, South Parks Road, Oxford OX1 3QR, United Kingdom \\ ${ }^{6}$ Department of Chemistry, University of Warwick, Gibbet Hill, Coventry, CV4 7AL,United Kingdom
}

(Received 11 October 2017; revised manuscript received 15 March 2018; published 5 April 2018)

\begin{abstract}
It is of great interest to design and make materials in which ferroelectric polarization is coupled to other order parameters such as lattice, magnetic, and electronic instabilities. Such materials will be invaluable in next-generation data storage devices. Recently, remarkable progress has been made in understanding improper ferroelectric coupling mechanisms that arise from lattice and magnetic instabilities. However, although theoretically predicted, a compact lattice coupling between electronic and ferroelectric (polar) instabilities has yet to be realized. Here we report detailed crystallographic studies of a perovskite $\mathrm{Hg}^{\mathbf{A}} \mathrm{Mn}_{3}^{\mathbf{A}^{\prime}} \mathrm{Mn}_{4}^{\mathbf{B}} \mathrm{O}_{12}$ that is found to exhibit a polar ground state on account of such couplings that arise from charge and orbital ordering on both the $\mathrm{A}^{\prime}$ - and B-sites, which are themselves driven by a highly unusual $\mathrm{Mn}^{\mathrm{A}^{\prime}}-\mathrm{Mn}^{\mathrm{B}}$ intersite charge transfer. The inherent coupling of polar, charge, orbital, and hence magnetic degrees of freedom make this a system of great fundamental interest, and demonstrating ferroelectric switching in this and a host of recently reported hybrid improper ferroelectrics remains a substantial challenge.
\end{abstract}

DOI: 10.1103/PhysRevB.97.144102

\section{INTRODUCTION}

Considerable efforts have been made in the search for novel multiferroics, and as such materials will undoubtedly find utility in future solid-state storage devices, driving both capacity and data access speeds ever higher. In these materials, it is essential to investigate the coupling between ferroelectric polarization and other order parameters [1-5]. Perovskites are a family of materials exhibiting a large number of technologically desirable physical properties. However, despite their very rich chemistry, ferroelectricity has been restricted to a rather narrow range of compositions, and there is good theoretical evidence to suggest that antipolar displacive instabilities generally act to suppress polar ones $[6,7]$. For the most part, experimental studies have hence been confined to $\mathrm{Ti}$-, $\mathrm{Pb}$-, or $\mathrm{Bi}$-containing perovskites, where a strong desire for these cations to undergo a second-order Jahn-Teller distortion provides a local driving force for the resulting proper ferroelectricity. On the other hand, in the field of improper ferroelectrics, remarkable progress has been made in understanding the coupling mechanisms that arise from lattice and magnetic instabilities [8-10]. For instance,

\footnotetext{
*m.senn@warwick.ac.uk

Published by the American Physical Society under the terms of the Creative Commons Attribution 4.0 International license. Further distribution of this work must maintain attribution to the author(s) and the published article's title, journal citation, and DOI.
}

recent work in the closely related layered Ruddlesden-Popper perovskites has led to theoretical predictions of improper ferroelectricity arising due to the coupling of two tilt modes of $\mathrm{BO}_{6}$ octahedra [2] to a third polar instability. However, for unlayered perovskites, it would appear that structural symmetry conspires to make such a coupling scheme utilizing tilt modes alone, impossible. Furthermore, despite theoretical predictions [11-14], a compact lattice coupling between electronic (orbital and charge degrees of freedom) and ferroelectric (polar) instabilities has yet to be experimentally realized. On the other hand, some perovskite materials are known to exhibit improper ferroelectricity by a so-called type-II multiferroic mechanism in which the polarization is a secondary order parameter of the primary magnetic ordering [15]. One of the most notable examples is $\mathrm{CaMn}_{7} \mathrm{O}_{12}$, which has a so-called 134-perovskite $\mathrm{AA}_{3}^{\prime} \mathrm{B}_{4} \mathrm{O}_{12}$ structure (top panel Fig. 1 for aristotype). This example was believed to represent the largest magnetically induced polarization reported to date $[16,17]$, although more recent findings dispute this [18]. This magnetoelectricity has been found to be associated with a magnetic ordering arising due to an unusual helical orbital order [19]. Despite the significant magnetoelectric response in this material, the ordering temperature remains low, as the mechanism still explicitly relies on the magnetic ordering, rather than just the orbital ordering which occurs at much higher temperatures. It is hence desirable to explore chemistries around $\mathrm{CaMn}_{7} \mathrm{O}_{12}$ and associated local degrees of freedom with the hope of coupling orbitally ordered states directly to ferroelectric properties. With the above in mind, we have synthesized the perovskite $\mathrm{HgMn}_{7} \mathrm{O}_{12}$ using high-pressure synthesis techniques. 

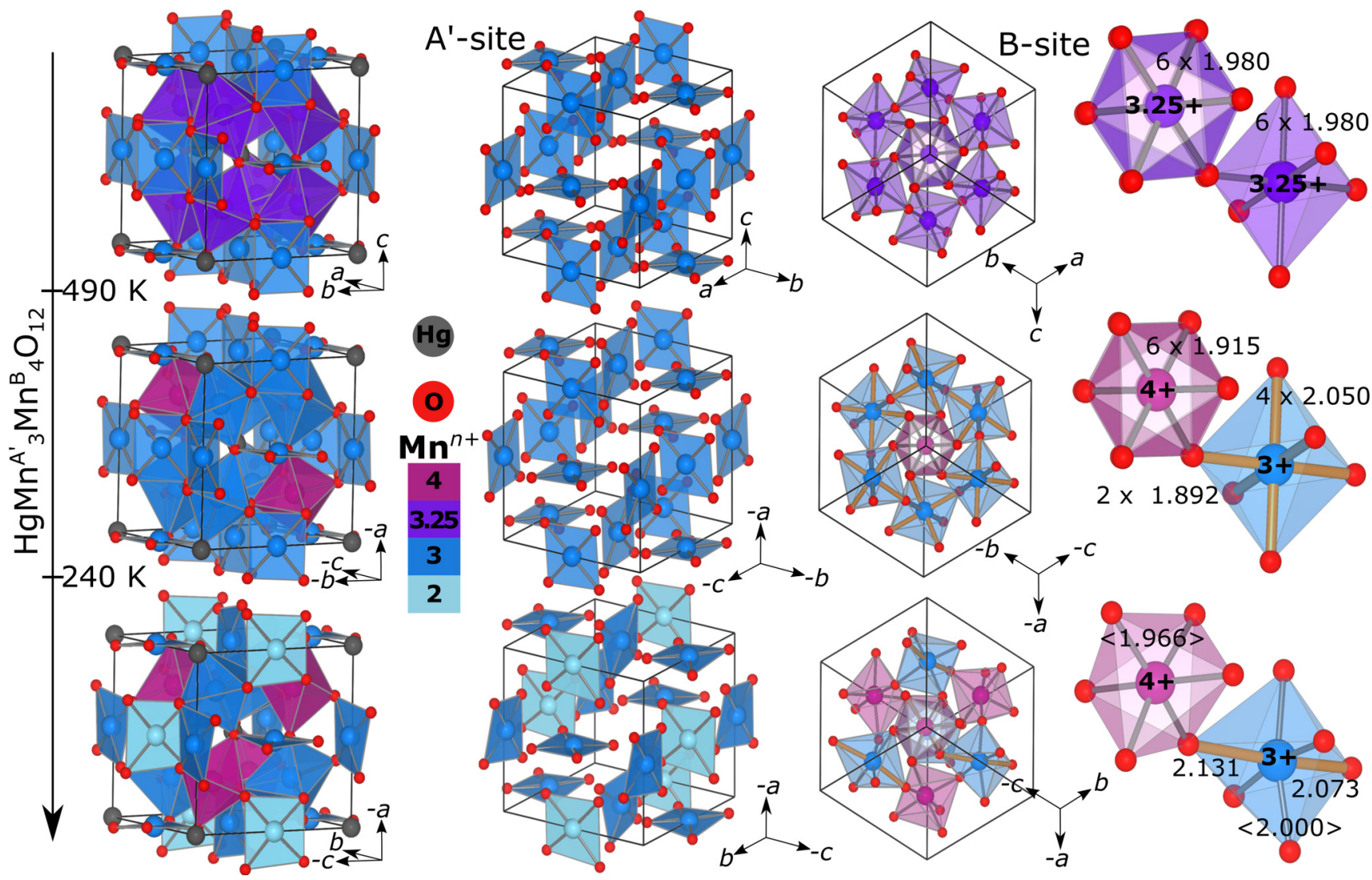

FIG. 1. The structure of the phases of $\mathrm{HgMn}_{7} \mathrm{O}_{12}$. Top panel, high-temperature cubic aristotype $\operatorname{Im} \overline{3}$; middle, $R \overline{3}$ orbital disorder phase; bottom, Pnn2, double charge-ordered, polar phase. The color key indicates the formal valence states of Mn sites assigned based on analysis of the crystal structure. The orange bonds represent the Jahn-Teller long bonds ( $d_{z}$ orbital order) of the $\mathrm{Mn}^{3+}$. The $R \overline{3}$ phase has four apparent Jahn-Teller long bonds in the unit cell which are actually an average of two short and two long bonds, observed on account of the orbital disorder. There is a charge transfer of one electron per unit cell from $\mathrm{B}$ - to $\mathrm{A}^{\prime}$-site on going from $R \overline{3}$ to Pnn2, which acts to remove the orbital disordered state and facilitates the double charge order on the $\mathrm{A}^{\prime}$ - and $\mathrm{B}$-site.

\section{EXPERIMENTAL DETAILS AND DATA ANALYSIS}

\section{A. Sample preparation}

Polycrystalline $\mathrm{HgMn}_{7} \mathrm{O}_{12}$ was prepared by solid-state reaction under high-pressure and high-temperature conditions. Stoichiometric amounts of $\mathrm{HgO}$ (Sigma-Aldrich $\geqslant 99.0 \%$ ), $\mathrm{MnO}_{2}$ (Alfa Aesar 99.997\%), and $\mathrm{Mn}_{2} \mathrm{O}_{3}$ (Aldrich 99.99\%) were well mixed and sealed in a platinum capsule. The capsule, a boron nitrite insulating layer, and a graphite heater were assembled in a pyrophyllite cell and placed in a DIA-type cubic anvil high-pressure apparatus. The sample was treated at $8 \mathrm{GPa}$ and $1600 \mathrm{~K}$ for $30 \mathrm{~min}$ and then released to ambient condition. Each synthesis produced a $\sim 0.1 \mathrm{~g}$ sample. Judging by $\mathrm{x}$-ray powder diffraction and magnetic susceptibility (see Supplementary Material (SM) [20], Fig. S1), products from 14 out of the 24 runs were selected and combined for further analysis in the neutron powder diffraction (NPD) studies.

\section{B. Crystallography and crystal structure analysis}

Full details of the refined average structures are given in the SM (Table S1) [20]. Synchrotron X-ray powder diffraction (SXRD) experiments were carried out for phase identification and crystal structure analysis. The powder sample was packed in a $0.1 \mathrm{~mm}$ borosilicate capillary tube to minimize the absorption effect and measured with a $15 \mathrm{keV}$ beam and the MYTHEN detector at beamline 09A, Taiwan Photon Source, and I11, Diamond Light Source, UK. NPD data on the combined $1.5 \mathrm{~g}$ polycrystalline sample was carried out at beamline Wombat [21] and Echidna [22], ANSTO, Australia. Temperature-dependent diffraction patterns were collected in a $6 \mathrm{~mm}$ diameter vanadium can and neutron wavelengths of 1.63 and $2.42 \AA$. The obtained data were analyzed within the Rietveld method using the program TOPAS, and refinements were done using the symmetry-adapted displacements formalism as parametrized by ISODISTORT [23] and implemented through the Jedit interface with TOPAS [24]. The parametrizations were all performed with respect to our high-temperature cubic structure $[\operatorname{Im} \overline{3},(550 \mathrm{~K})]$, with a setting where $\mathrm{Hg}$ sits on $(0,0,0) ; \mathrm{A}^{\prime} \mathrm{Mn} 1(0,0.5,0.5)$; B Mn2 $(0.25,0.25,0.25)$, and $\mathrm{O}(x, y, 0)$. For the rhombohedral phase (room temperature), in order to facilitate an easy comparison between this, the parent structure and the orthorhombic phase, we adopted here a $R \overline{3}$ rhombohedral setting which retains the parent unit-cell axes $[a=7.37987 \AA, \alpha=90.37922$, $[(0,0,-1),(0,-1,0),(-1,0,0)]$, origin $=(0,0,0)]$, applying the appropriate symmetry constraints. Selected SXRD and NPD patterns, and analysis results are shown in Figs. 2 and 3. 

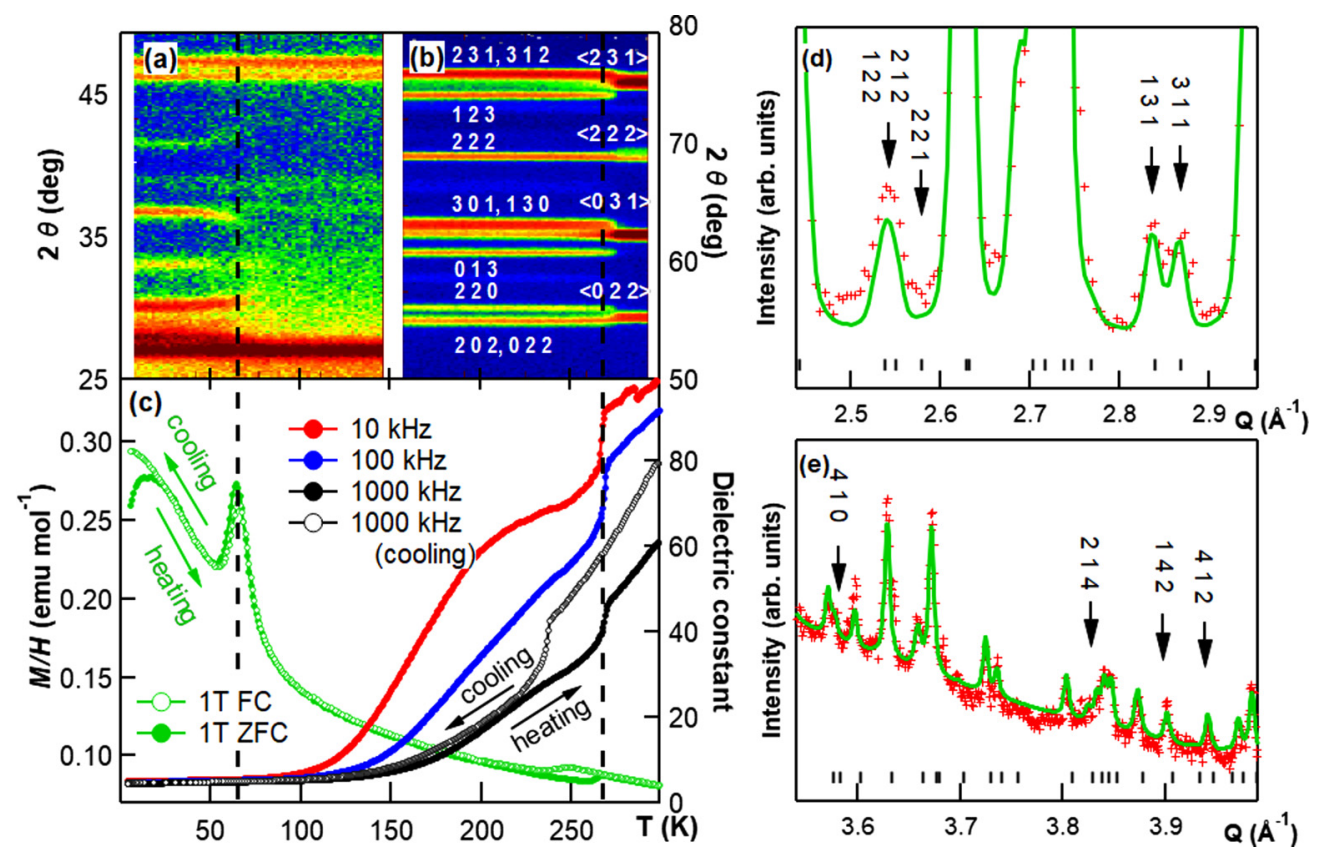

FIG. 2. Structural and physical property characterization of $\mathrm{HgMn}_{7} \mathrm{O}_{12}$. (a, b) Selected peaks from the neutron diffraction as a heat map, showing both the pronounced lattice distortion which occurs at $260 \mathrm{~K}$ on warming ( $240 \mathrm{~K}$ on cooling) and the low-temperature magnetic phase transition at $60 \mathrm{~K}$. (c) Field- and zero-field-cooled magnetic susceptibility taken with a measuring field of $1 \mathrm{~T}$ and a dielectric susceptibility measured at three different frequencies as indicated. (d, e) Weak superstructure reflections fitted with the Pnn2 model in Echidna NPD and I1 1 SXRD patterns, respectively.

Careful examination of the neutron diffraction data at $100 \mathrm{~K}$ revealed additional peaks which violate the body-centering of the parent $\operatorname{Im} \overline{3}$ cell [Fig. 2(d)]. Only very weak signatures of these additional reflections could be found in the highresolution SXRD data [Fig. 2(e)], indicating that they most likely arise due to subtle distortions of the oxygen atoms. In the propagation vector formalism, these additional weak reflections can be indexed with $\mathbf{k}=[1,1,1]$ (H-point). In a similar spirit to the exhaustive approach for structural determination employed recently in the case of the phase transitions in $\mathrm{Bi}_{2} \mathrm{Sn}_{2} \mathrm{O}_{7}$ [25], we generated a complete list of possible structural models which are necessarily subgroups of the parent symmetry $\operatorname{Im} \overline{3}$ and are compatible with both the observations of metric orthorhombic symmetry and the occurrence of reflections that can be indexed on $\mathbf{k}=[1,1,1]$. In total we tested 10 models and, while many gave near equivalent fitting statistics to the combined neutron and synchrotron $\mathrm{x}$-ray diffraction $\mathrm{Ri}$ etveld refinements that we performed on data collected at 100 K, only the Pnnm and Pnn2 models provided a satisfactory fit to the weak superstructure reflections [see Figs. 2(d) and 2(e)]. To facilitate a discrimination between these models, several grains of $\sim 10$ micron diameter were selected from the powder for single crystal diffraction data collections at 100-120 K. The single crystal data was measured on the I19 beamline, Diamond Light Source equipped with a Pilatus $2 \mathrm{M}$ detector. The measurements were performed with a wavelength of $0.6889 \AA$. Single crystal Bragg intensities were integrated using the program XDS [26]. Since XDS does not allow multirun experiments to be processed, all runs were integrated separately by manually transforming the crystal orientation matrix and then manually scaling them together. A combined refinement in TOPAS against this single crystal data and the neutron and $\mathrm{x}$-ray powder diffraction data clearly show that the polar space group Pnn2 $[a=7.22271(3) \AA, \quad b=7.32475(3) \AA, c=$ $7.58504(3) \AA$, $[(0,1,0),(0,0,-1),(-1,0,0)]$, origin $=(0,0,0)]$ provides a significantly better fit to the data (see SM [20], Tables S2 and S3, and Fig. S2). The reciprocal space reconstructions presented here (Figs. 4 and 5) are from two different measurements taken at $120 \mathrm{~K}$.

\section{Magnetic and transport property measurements}

Magnetic property measurements were carried out with a commercial magnetometer, Quantum Design Magnetic Properties Measurement System (VSM-MPMS). Zero-field-cooled and field-cooled magnetic susceptibility were measured at $5-300 \mathrm{~K}$ in an external magnetic field of $10 \mathrm{kOe}$. The dielectric constant measurements were made using the Agilent 4294A precision impedance analyzer by applying an ac voltage of $1 \mathrm{~V}$. For pyroelectric current measurements, an electrical poling field was applied during the cooling down to base temperature, and pyroelectric current was obtained upon heating using a Keithley 6517B electrometer.

Temperature-dependent dielectric constant measurements of $\mathrm{HgMn}_{7} \mathrm{O}_{12}$ with a range of frequencies from $10 \mathrm{kHz}$ to $1 \mathrm{MHz}$ are presented in Fig. 2(c), exhibiting a frequencyindependent steplike anomaly near the structural transition. The long-range ordering represents a thermal hysteresis behavior, which could be associated with magnetization curves. Based on the Rietveld analysis of high-resolution SXRD patterns, $\mathrm{HgMn}_{7} \mathrm{O}_{12}$ undergoes a structural transition from centrosymmetric $R \overline{3}$ to noncentrosymmetric Pnn2 with a 

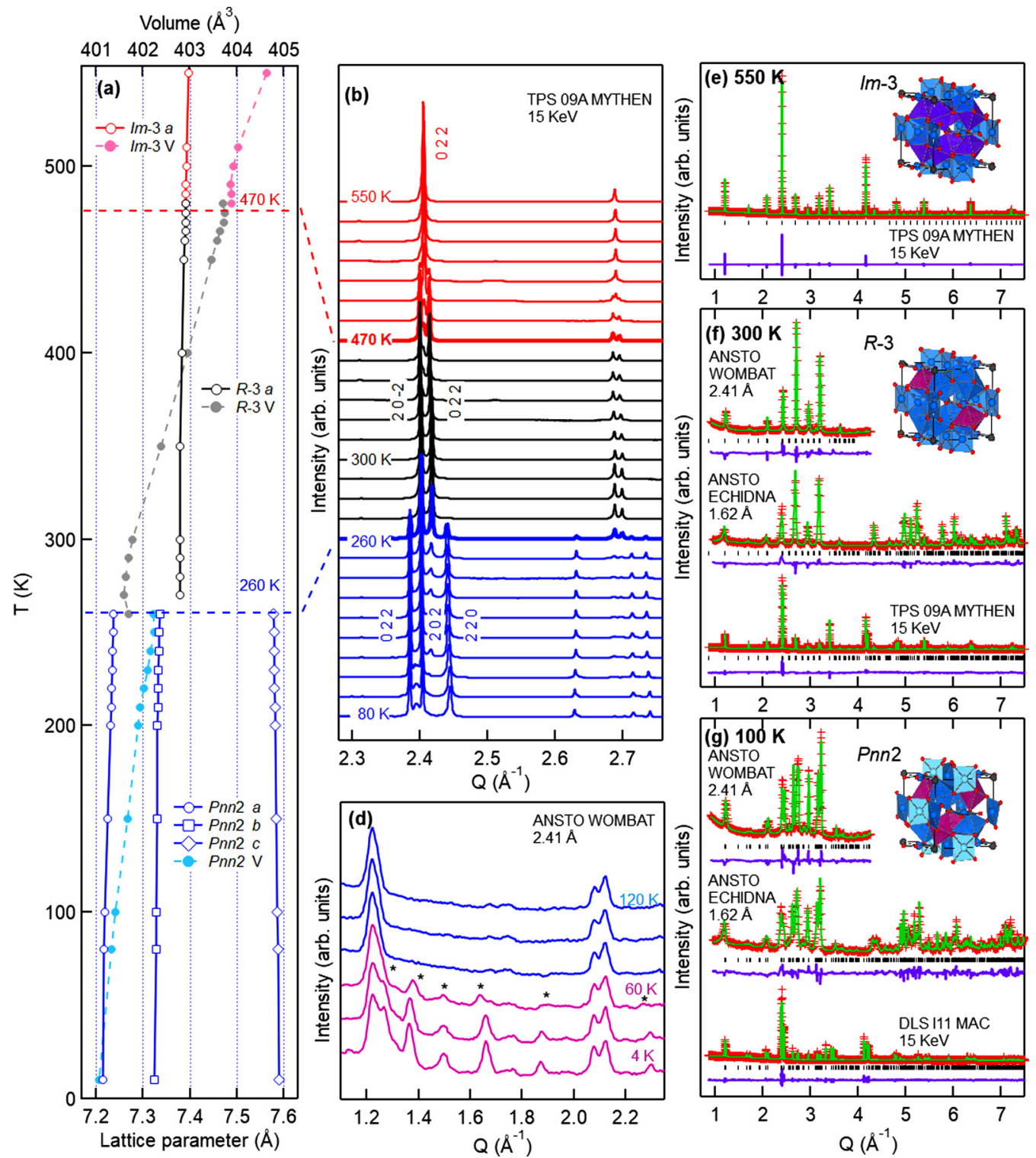

FIG. 3. (a) Temperature evolution of lattice parameters of $\mathrm{HgMn}_{7} \mathrm{O}_{12}$ phases. (b, c) Selected $Q$ range of SXRD data showing the lattice splitting and phase evolution of $\mathrm{HgMn}_{7} \mathrm{O}_{12}$. (d) Selected $Q$ range of NPD data showing the development of long-range magnetic ordering. (e)-(g) Joint Rietveld refinement results of SXRD and NPD data. The insets show the crystal structure of $\mathrm{HgMn}_{7} \mathrm{O}_{12}$ phases.

polar structure, which invokes charge ordering between the $\mathrm{Mn} \mathrm{A}^{\prime}$-site and the Mn B-site. The results suggest that the frequency-independent steplike anomaly could be an improper ferroelectric like ordering. In addition, there is also a frequency-independent peak close to $T_{N}$. For investigating the possibility of an additional ferroelectric component in $\mathrm{HgMn}_{7} \mathrm{O}_{12}$ at lower temperatures, temperature-dependent pyrocurrent measurements with different applied electric fields and heating rates were performed (see Fig. 6). The reliable pyrocurrent peaks with different applied electric fields 0,1 , and $2 \mathrm{kV} / \mathrm{cm}$ and different heating rates 5 and $10 \mathrm{~K}$ per min are displayed in Fig. 6(b). A switchable pyrocurrent peak near $T_{N}$ through a poling electric field of $\pm 1 \mathrm{kV} / \mathrm{cm}$ is represented in inset of Fig. 6(c), signifying the occurrence of an additional ferroelectric component below $T_{N}$.

\section{RESULTS AND DISCUSSION}

\section{A. Structural phase transitions}

Our variable-temperature neutron and synchrotron $\mathrm{x}$ ray powder diffraction and physical property measurements (Fig. 2) reveal three distinct structural phase transitions that we will focus on in this paper. On cooling an additional low-temperature $(63 \mathrm{~K})$ magnetic transition was observed, showing an incommensurate magnetic ordering nature. The magnetic susceptibility and isothermal magnetization results indicate a soft ferrimagnetic behavior of the compound. Details of the modulated magnetic structure will be discussed further elsewhere. Above $490 \mathrm{~K}$ the $\operatorname{Im} \overline{3}$ aristotype is observed (Fig. 1). On cooling, a first-order phase transition is evident via a phase coexistence in the high-temperature SXRD data 
between 470 and $490 \mathrm{~K}$ (Fig. 3), and the structure can be indexed on the same rhombohedral space group reported for the charge-ordered room-temperature structure of $\mathrm{CaMn}_{7} \mathrm{O}_{12}$ [27].

At room temperature, we find a 1:3 charge-ordered state of the Mn B-sites, with bond valence sums (BVS) extracted from our Rietveld refinements of 3.63+:3.24+, consistent with previous reports for $\mathrm{CaMn}_{7} \mathrm{O}_{12}$ [27]. We consider the formal valence states to be $4+$ and $3+$, and the magnitudes of the charge segregation as observed by BVS calculations are typical of those commonly found in other metal oxides [28]. Further analysis of the crystal structure reveals that the $\mathrm{Mn}^{3+}$ sites exhibit the unusual Jahn-Teller compression (two short, four long $\mathrm{Mn}-\mathrm{O}$ bonds) rather than the elongation (two long, four short) expected for $\mathrm{Mn}^{3+}\left(t_{2 g}^{3} e_{g}^{1}\right)$ in an octahedral crystal field. Invariably, such observations can be attributed to orbital disorder [29]. Here, we attribute the two short bonds being ordered along the unique rhombohedral axis, with a disordering between two short and two long bonds in the perpendicular plane (Fig. 1), leading to a scenario where in the average crystallographic structure two short and four medium bond lengths are observed.

On cooling below $240 \mathrm{~K}$, another crystallographic phase transition is evident by a dramatic orthorhombic lattice distortion [Fig. 2(b)]. Hysteresis in both the magnetic and dielectric susceptibilities [Fig. 2(c)] indicates that the phase transition is strongly first order. The large change in the dielectric constant suggests a substantial structural change has occurred at the phase transition. Of the ten possible orthorhombic subgroups of $\operatorname{Im} \overline{3}$ consistent with the observed propagation vector $\mathbf{k}=$ $[1,1,1]$ (H-point), only a model in $P n n 2$ provided a satisfactory fit simultaneously to the weak superstructure reflections in the powder diffraction data [Figs. 2(d) and 2(e)] and the single crystal data collected on grains taken from the polycrystalline samples (Fig. 4).

\section{B. Diffuse scattering and structural modulations}

Additional features in the single crystal diffraction data, some that evolve over time below $240 \mathrm{~K}$, reveal that further very small modulation to the average crystallographic structure presented above may occur as a competing kinetic phase. Although our discussion in the rest of this paper will focus on the average crystallographic results, we present here for completeness all additional observed features, however subtle they may be.

Figure 4 shows the $h k 0, h 0 l$, and $0 k l$ planes of reciprocal space reconstructed from a small (radius 5 microns) crystal of $\mathrm{HgMn}_{7} \mathrm{O}_{12}$ at $100 \mathrm{~K}$ from data taken at I19, Diamond Light Source, showing violations of systematic absence conditions imposed by the $n$-glide (arrows) and $2_{1}$ screw axes (insets). The violations are most evident for $P 11 n$ [indicated by arrows in Fig. 4(a)] in the $h k 0$ plane, and hence our initial assignment of space group Pnnm or Pnn2 symmetry based on the powder diffraction data alone can be explained. Refinements against the integrated structure factors extracted against this data and those collected on several other crystals reveal that the Pnn2 space group provides a significantly better fit to the data than the centrosymmetric Pnnm model, and this is the basis of our final assignment of the average structure discussed above
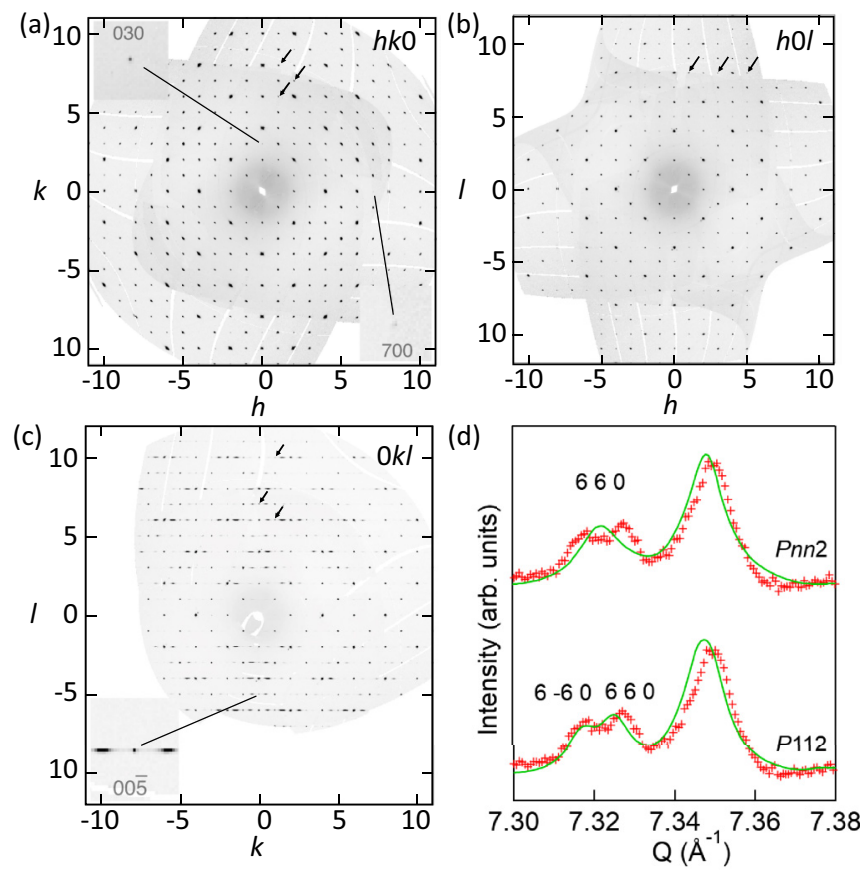

FIG. 4. Planes of reciprocal space reconstructed from small (radius 5 microns) crystal of $\mathrm{HgMn}_{7} \mathrm{O}_{12}$ at $100 \mathrm{~K}$ from data taken at I19, Diamond Light Source, showing violations of systematic absence conditions, (a) $h k 0$, (b) $h 0 l$, and (c) $0 k l$, and I11 synchrotron powder diffraction data (d) evidencing a slight monoclinic splitting.

(see SM [20] Fig. S2, and Tables S2 and S3). Additional further violations of $P 1 n 1$ and $P n 11$ glides are indicated by arrows in Figs. 4(b) and 4(c), but these are at least an order of magnitude weaker than the $P 11 n$ violations (which are themselves already very weak). The insets also show a violation for the $P 12{ }_{1} 1$ axes and exceptionally weak violations $P 2{ }_{1} 11$, which taken together with the much stronger $P 112_{1}$ violations [inset of Fig. 4(c)] would suggest a space group of $P 222$ or $P m m m$. However, neither of these are a subgroup of $P n n 2$ or $P n n m$, which were the only orthorhombic models providing a satisfactory fit to the powder diffraction data, and we must hence conclude that the true average crystallographic symmetry is no higher than $P 112$ in the present setting. There is some evidence for splitting of the high angle peaks $(h+k \geqslant$ 10) of the I11 synchrotron powder diffraction data Fig. 4(d) supporting a $P 112$ model, but we estimate that this splitting is less than $\gamma<90.06^{\circ}$. Refinements in this space group neither significantly improve the fitting statistic (see SM [20], Table S4) nor change the physical interpretation of the model, and so our analysis in this paper focuses on the Pnn2 model.

Evolution of diffuse scattering below the phase transition at $260 \mathrm{~K}$ is shown at $120 \mathrm{~K}$ from overexposed diffraction data in Fig. 5(a) reconstructed from a quickly cooled crystal and after $80 \mathrm{~min}$ at $120 \mathrm{~K}$ in Fig. 5(b). In addition to the Bragg peaks violating the $I$-centering condition $h+k+l=$ even discussed above, these patterns show some other features. Right after the phase transition, the crystals develop additional streaks of diffuse scattering along the $b^{*}$-axis which can also be seen in the inset of Fig. 4(c). The intensity of the streaks occurs with maxima at approx. $h, k \pm 0.53, l$ (see insets in Fig. 5). 

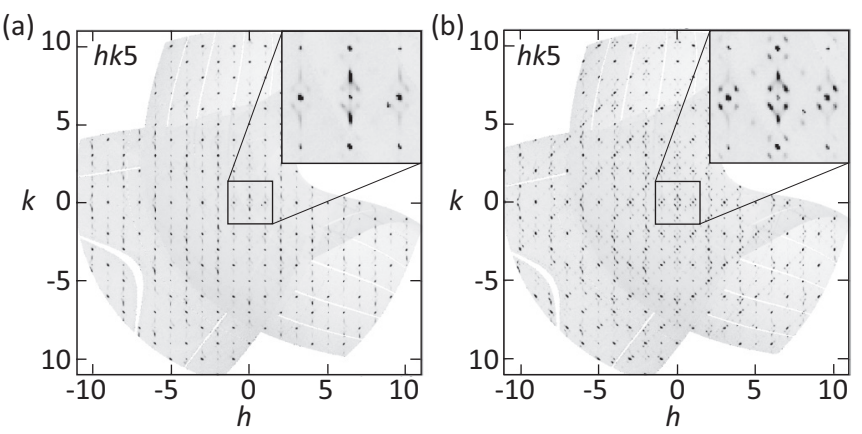

FIG. 5. Evolution of diffuse scattering below the phase transition at $260 \mathrm{~K}$, here shown at $120 \mathrm{~K}$ from overexposed diffraction data: (a) reconstruction on a quickly cooled crystal and (b) after $80 \mathrm{~min}$ at $120 \mathrm{~K}$.

The intensity of this diffuse scattering gradually decreases as $l$ tends to zero and is absent from the $h k 0$ plane. This is very characteristic for crystals with displacive disorder. In our case it means that part of the motion which is captured by the atomic displacement parameters has static rather than dynamic origin. In real structures, these displacements form a highly correlated pattern in the $a c$ plane, while along the $c$-axis the correlations decay away very quickly. Additionally, the fact that the maxima of diffuse scattering lies at a low-symmetry position in reciprocal space means that the local order along the $b$-axis has an incommensurate nature. In addition to the diffuse streaks, at low temperature after some time, further features in the diffraction pattern develop. These can be characterized as satellite reflections with the modulation vectors $\mathbf{k}=\left[\frac{1}{6}\right.$, $\left.\frac{1}{6}, 0\right]$ and $\left[\frac{1}{6},-\frac{1}{6}, 0\right]$ and another set of streaks which form a Y-shaped pattern with the streaks along the $b^{*}$-axis (see insets). A possible interpretation of these features would be that the crystal is slowly turning to a commensurately modulated lower temperature phase and that the diffuse scattering streaks are formed by the domain walls between the disordered phase and low temperature modulated phase. The significance of diffuse scattering and disorder in canonical orbital ordered/disordered systems such as $\mathrm{LaMnO}_{3}$ remains of great interest [30], and a fuller interpretation of our findings in this 134-perovskite, partially doped system will form a substantial piece of future work.

\section{Charge ordering and improper ferroelectric coupling}

We now give a full analysis of the average low temperature structure of $\mathrm{HgMn}_{7} \mathrm{O}_{12}$, showing that the phase transition is driven by intersite charge transfer, followed by charge ordering on both the B- and $\mathrm{A}^{\prime}$-sites. Inspection of the crystal structure reveals that the formally equivalent $\mathrm{A}^{\prime}$-site in the rhombohedral phase is now split into three Wyckoff sites of equal multiplicity. Furthermore, the B-sites, which were split across two sites of 1:3 ratio in multiplicity, now occupy two sites of equal multiplicity. The BVS on these 1:1 split B-sites are $3.17+: 3.41+$, and are hereafter referred to formally as $3+$ and $4+$. This assignment is further validated by the evidence of Jahn-Teller elongations (two long, four short bonds) present in the formal $\mathrm{Mn}^{3+}$ state and absent in $\mathrm{Mn}^{4+}$ (Fig. 1). The above description with an average B-site Mn valence of 3.5+ requires either an average formal valence state change of $\mathrm{Mn}^{3+} \rightarrow \mathrm{Mn}^{2.667+}$ on the $\mathrm{A}^{\prime}$-site or a valance state change of $\mathrm{Hg}^{2+} \rightarrow \mathrm{Hg}^{1+}$ to achieve charge neutrality. $\mathrm{Hg}^{2+} \rightarrow \mathrm{Hg}^{1+}$ on cooling through the phase transition can be discounted, as the BVS actually shows a small increase from $1.89+(R \overline{3})$ to $2.12+(P n n 2)$ on cooling, rather than a decrease expected by this scenario. Additionally, $\mathrm{Hg}^{1+}$ compounds are generally only stabilized by $\mathrm{Hg}$ dimers, and to the best of our knowledge there are no reports in the literature of stable metal oxides with undimerized (i.e., paramagnetic) $\mathrm{Hg}(\mathrm{I})$ ions. On the other hand, $\mathrm{Mn}^{2+}$ in the square-planar coordination geometry is known and has been reported for a number of closely related perovskite systems [31-33]. Based on crystal field stabilization arguments, $\mathrm{Mn}^{2+}$ in the square-planar environment tends to restore the expected orbital degeneracy preferred by the symmetrically filled $e_{g}$ and $t_{2 g}$ orbitals by making its crystal field environment more regular by shortening second-nearestneighbor Mn-O bond distances. Such a shortening is evident in our low temperature refinements for $\mathrm{Mn}^{\mathrm{A}^{\prime}}$, which have a nextnearest-neighbor distance of $2.4 \AA$, very close to that reported in other systems with $\mathrm{Mn}^{\mathrm{A}^{\prime 2} 2+}[31,32]$, and is well below the shortest second-nearest-neighbor distance of $2.65 \AA$ Abserved for any previously reported $\mathrm{Mn}^{\mathrm{A}^{\prime} 3+}$ system. We hence assign a formal charge ordering of $1: 2, \mathrm{Mn}^{2+}: \mathrm{Mn}^{3+}$ on the $\mathrm{A}^{\prime}$-site.

This scenario of charge transfer and charge ordering is further supported by the observations of volume expansion (negative thermal expansion) on going from the high temperature rhombohedral phase to the low temperature $P n n 2$ phase, a phenomena which is observed in other 134-perovskite charge-transfer systems [34]. Based on crystal ionic radii, the charge transfer and associated change in formal valence states is calculated to give $0.5 \AA^{3}$ volume change per unit cell, in excellent agreement with the $0.4 \AA^{3}$ experimentally observed (Fig. 3). Such intersite charge transfer is rare but is known to act to remove unstable valence states such as in $\mathrm{Fe}^{4+}$ containing perovskite, $\mathrm{LaCu}_{3} \mathrm{Fe}_{4} \mathrm{O}_{12}$ [34]. In $\mathrm{HgMn}_{7} \mathrm{O}_{12}$, the integer intersite transfer is between two sites which are of the same element but have different coordinations. We believe this to be the first example of temperature-induced charge transfer in the manganites. It seems probable that this process acts to remove the frustration associated with the orbital disordered $R \overline{3}$ phase.

The atomic displacements arising due to the charge and orbital order on the B-sites in the Pnn2 phase were decomposed in ISODISTORT as transforming as the zone-boundary $\mathbf{k}=[1,1,1]$ irreducible representations $\mathrm{H}_{1}^{-}$and $\mathrm{H}_{2}^{-} \mathrm{H}_{3}^{-}$of the parent $\operatorname{Im} \overline{3}$ phase. The irreducible representations are tabulated within ISODISTORT, and their characters are included in the SM [20] (see Table S5). A single order parameter transforming as $\mathrm{H}_{2}^{-} \mathrm{H}_{3}^{-}$is sufficient to induce the observed orthorhombic distortion and drive the aforementioned charge and orbital order on the B-site. However, the action of this order parameter lowers the space group symmetry only as far as Pnnn, and an additional primary order parameter transforming as $\mathrm{H}_{4}^{+}$is required to lower the symmetry to the observed Pnn2. The role of the additional degrees of freedom in the Pnn2 model (transforming as $\mathrm{H}_{4}^{+}$) is to help to restore the orbital degeneracy on the $\mathrm{A}^{\prime}$-site $\mathrm{Mn}^{2+}$ by making the coordination environment more regular and shortening the second-nearest-neighbor distance (Fig. 7). As both these lattice modes are necessary and sufficient to lower the symmetry of 

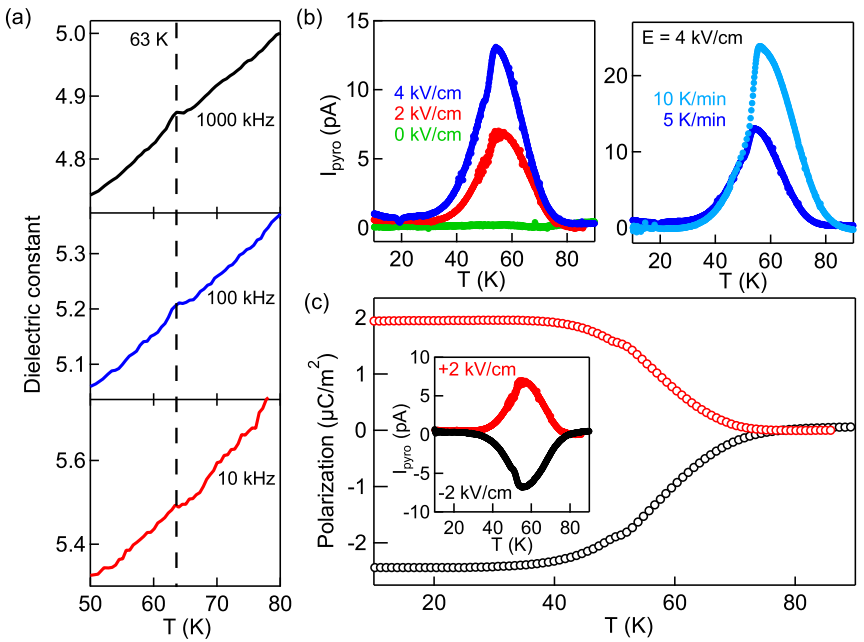

FIG. 6. Electrical results for polarization induced by the magnetic ordering transition. (a) Anomaly at magnetic transition temperature shown in dielectric constant at various frequencies. (b) Pyrocurrent measured at various electric fields and temperature ramping speeds. (c) A switchable pyrocurrent peak and polarization through a poling electric field of $\pm 2 \mathrm{kV} / \mathrm{cm}$ between 10 and $90 \mathrm{~K}$.

centrosymmetric $\operatorname{Im} \overline{3}$ to the polar space group $P n n 2$, there must exist a coupling to a third secondary ferroelectric order parameter. The trilinear term responsible for the improper ferroelectric polarization discussed in this paper is hence $\mathrm{H}_{2}^{-} \mathrm{H}_{3}^{-} \mathrm{H}_{4}^{+} \mathrm{P}$. We note that another possibility would be that polarization and one of the two zone-boundary modes forms the primary order parameter. However, this scenario would lead to a divergence in the experimentally measured dielectric constant rather than the steplike transition (Fig. 2) observed in the present work, which is more consistent with an improper ferroelectric mechanism [1]. Unfortunately, the measurements of pyrocurrent or polarization-electric field (PE) loops in the Pnn2 phase at higher temperatures above the magnetic ordering transition has been impossible due to leakage currents at these higher temperatures. Obtaining such direct measures of the switchable nature of the crystallographically identified polar ground state is a substantial future challenge for this and other recently reported hybrid improper ferroelectrics $[2,35]$. Our following discussion on ferroelectric switching pathways is based on symmetry arguments alone.

Assuming a spontaneous charge transfer and ordering on the $\mathrm{A}^{\prime}$ - and B-sites, an improper polarization is hence always expected to arise as a secondary order parameter and will be linearly dependent on the magnitude of the charge and orbital order on the $\mathrm{A}^{\prime}$ - and B-sites. Additionally, the switching of the direction of the polarization (Fig. 7) will proceed by either a reversal of the checkerboard charge ordering on the B-site or by a reversal of a lattice mode that influences the $\mathrm{A}^{\prime}$-site charge ordering. Calculation of the polarization from our Pnn2 model in the point charge approximation using average formal valence states for $\mathrm{A}^{\prime}$ - and $\mathrm{B}$-sites and our crystallographic coordinates reveals a theoretical spontaneous polarization of $8 \mu \mathrm{C} \mathrm{cm}^{-1}$ along the crystallographic $c$-axis. Such calculations on the topical improper ferroelectric $\mathrm{Ca}_{3} \mathrm{Ti}_{2} \mathrm{O}_{7}\left(16 \mu \mathrm{C} \mathrm{cm}^{-1}\right)$ [36] have been found to give a reasonable estimate for the experimental switchable polarizations $\left(8 \mu \mathrm{Ccm}^{-1}\right)$ [37] and

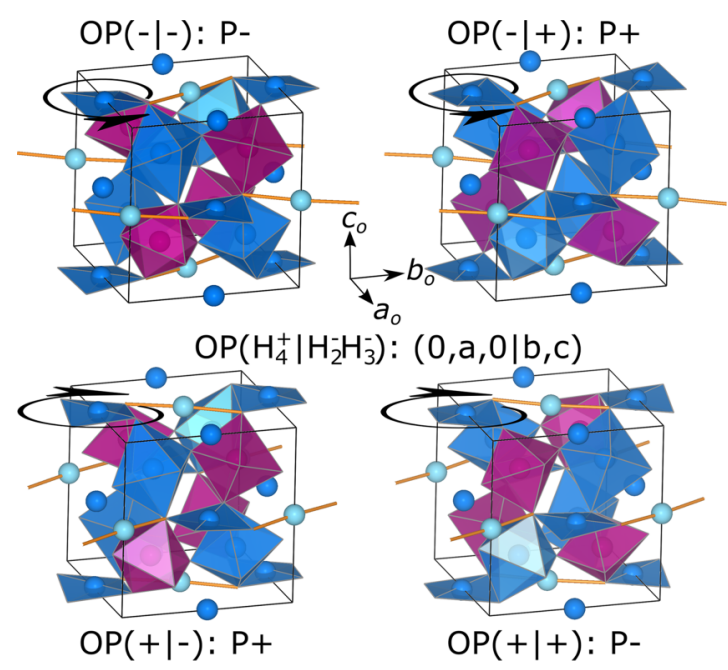

FIG. 7. The origin of the improper polarization in $\mathrm{HgMn}_{7} \mathrm{O}_{12}$. The reversal of one or the other of the two primary order parameters responsible for the double-charge ordering $\left(\mathrm{H}_{4}^{+}\right.$and $\left.\mathrm{H}_{2}^{-} \mathrm{H}_{3}^{-}\right)$on the $\mathrm{A}^{\prime}$ and $\mathrm{B}$-sites is sufficient to reverse the direction of the polarization $(\mathrm{P})$; reversal of both order parameters returns the polarization to its initial state. The short second-nearest-neighbor Mn-O distance $(\sim 2.4 \AA)$ that is indicative of the $\mathrm{Mn}^{\mathrm{A}^{\prime} 2+}$ state is shown in yellow (square-planar nearest-neighbor coordination omitted for clarity).

are indeed comparable to what we find here from our point charge calculations.

There has been a large amount of renewed interest in improper ferroelectric mechanisms recently, particularly in studying the coupling between two nonpolar zone-boundary lattice modes with octahedral tilt character in the RuddlesdenPopper layered perovskites, which has been discussed in the context of "hybrid improper ferroelectricity" [2,35-37]. Here we reveal a mechanism by which two zone-boundary modes, implicitly dependent on orbital degrees of freedom, combine to give polar zone-centered displacements. These two modes should be strongly "hybridized" with each other, as charge ordering on $\mathrm{A}^{\prime}$ - and B-sites most likely occurs simultaneously rather than stepwise.

There are a few reports of other systems exhibiting polar distortions on account of charge or orbital ordering; however, many of these remain highly contentious [5,38]. In the halfdoped manganites, a combination of bond-centered and sitecentered charge orderings may give rise to ferroelectricity [4]. More recently, it has been proposed that in cation ordered $\mathrm{SmBaMn}_{2} \mathrm{O}_{6}$, that a combination of perovskite tilting and charge ordering drives a polar ground state [14]. $\mathrm{In}_{\mathrm{LuFe}} \mathrm{O}_{4}$, due to geometric frustration associated with charge ordering on a triangular lattice, a layered charge ordering leading with a net polarization may occur [39]. While in $\mathrm{Fe}_{3} \mathrm{O}_{4}$, the canonical example of a charge-ordered system, the resulting pattern of the orbital ordering alone breaks inversion symmetry, leading to polar distortions. However, the low-temperature structure is incredibly complex and can accurately be described only by the superposition of a very large number of lattice modes [40]. Our present work on $\mathrm{HgMn}_{7} \mathrm{O}_{12}$, on the other hand, demonstrates a compact and elegant coupling of orbital degrees of freedom directly to ferroelectric polarization. 


\section{Magnetoelectric coupling}

It is worth noting that as the underlying magnetic structure is explicitly reliant on the nature of the orbital and charge ordering in these systems, any coupling between ferroelectricity and magnetism in the magnetically ordered ground state is expected to be very strong. Indeed, we see experimental evidence for a dielectric anomaly (Fig. 6) at the magnetic ordering transition, and our pyrocurrent measurements suggest an additional type-II contribution to the polarization that is at least an order of magnitude larger than recently measured in $\mathrm{CaMn}_{7} \mathrm{O}_{12}$ [18]. It is important to note that our pyrocurrent results are for the polarization induced by the magnetic ordering transition only and do not pertain to the high-temperature polarization discussed in the manuscript that was calculated from the crystal structure in the point charge approximation only and is several orders of magnitude larger. A detailed understanding of the coupling terms involved in this additional low-temperature contribution to the improper ferroelectric polarization will require knowledge of the low-temperature magnetic structure, and this investigation will be undertaken as part of a separate communication.

\section{CONCLUSION}

We have demonstrated experimentally how a relatively simple charge and orbital ordering pattern can lead to a polar structure via an improper ferroelectric mechanism that involves the coupling between two zone-boundary lattice instabilities, to a third polar one. Our results represent a different realiza- tion of schemes coupling electronic and ferroelectric order parameters together that have recently been discussed in $a b$ initio studies on $\mathrm{A}^{\prime} \mathrm{AB}_{2} \mathrm{O}_{6}$ half-doped perovskites $[11,12,14]$. As the lattice instabilities that we report are directly linked to charge and orbital degrees of freedom on the $\mathrm{A}^{\prime}$ - and $\mathrm{B}$-sites, the coupling between electronic and ferroelectric order parameters is expected to be very strong. This opens up a possible route for electronic control of Jahn-Teller distortions and orbitally ordered states, an appealing prospect given the numerous technologically relevant properties that depend on these fundamental orderings.

\section{ACKNOWLEDGMENTS}

This work was supported by a joint International Exchange Scheme between Royal Society, UK (Grant No. IE141335) and Ministry of Science and Technology (MOST), Taiwan (Grants No. 104-2911-I-002-535, and No. 105-2911-I-002-515). The synchrotron beam time used in this paper was at I11 through the Diamond Light Source Block Allocation Group award "Oxford Solid State Chemistry BAG to probe compositionstructure-property relationships in solids" (EE13284), and at I19 (mt13639 and mt15920), and at 09A, Taiwan Photon Source, National Synchrotron Radiation Research Center (2016B0091 and 2017-1-127-1), and at ID22, European Synchrotron Radiation Facility (HC2331). Neutron powder diffraction was performed at Wombat (P4864) and Echidna (MI5930), ANSTO. M.S.S. would like to acknowledge the Royal Commission for the Exhibition of 1851 and the Royal Society for fellowships, and W.-T.C. thanks MOST for financial support (Grant No. 103-2112-M-002-022-MY3).
[1] E. Bousquet, M. Dawber, N. Stucki, C. Lichtensteiger, P. Hermet, S. Gariglio, J.-M. Triscone, and P. Ghosez, Improper ferroelectricity in perovskite oxide artificial superlattices, Nature (London) 452, 732 (2008).

[2] N. A. Benedek and C. J. Fennie, Hybrid Improper Ferroelectricity: A Mechanism for Controllable Polarization-Magnetization Coupling, Phys. Rev. Lett. 106, 107204 (2011).

[3] W. Eerenstein, N. D. Mathur, and J. F. Scott, Multiferroic and magnetoelectric materials, Nature (London) 442, 759 (2006).

[4] D. V. Efremov, J. van den Brink, and D. I. Khomskii, Bondversus site-centred ordering and possible ferroelectricity in manganites, Nat. Mater. 3, 853 (2004).

[5] J. van den Brink and D. I. Khomskii, Multiferroicity due to charge ordering, J. Phys.: Condens. Matter 20, 434217 (2008).

[6] N. A. Benedek and C. J. Fennie, Why are there so few perovskite ferroelectrics? J. Phys. Chem. C 117, 13339 (2013).

[7] U. Aschauer and N. A. Spaldin, Competition and cooperation between antiferrodistortive and ferroelectric instabilities in the model perovskite $\mathrm{SrTiO}_{3}$, J. Phys.: Condens. Matter 26, 122203 (2014).

[8] A. B. Harris, Landau analysis of the symmetry of the magnetic structure and magnetoelectric interaction in multiferroics, Phys. Rev. B 76, 054447 (2007).

[9] P. Barone and S. Picozzi, Mechanisms and origin of multiferroicity, C. R. Phys. 16, 143 (2015).
[10] N. A. Benedek, J. M. Rondinelli, H. Djani, P. Ghosez, and P. Lightfoot, Understanding ferroelectricity in layered perovskites: New ideas and insights from theory and experiments, Dalton Trans. 44, 10543 (2015).

[11] N. C. Bristowe, J. Varignon, D. Fontaine, E. Bousquet, and P. Ghosez, Ferromagnetism induced by entangled charge and orbital orderings in ferroelectric titanate perovskites, Nat. Commun. 6, 6677 (2015).

[12] J. Varignon, N. C. Bristowe, E. Bousquet, and P. Ghosez, Coupling and electrical control of structural, orbital and magnetic orders in perovskites, Sci. Rep. 5, 15364 (2015).

[13] A. Stroppa, P. Barone, P. Jain, J. M. Perez-Mato, and S. Picozzi, Hybrid improper ferroelectricity in a multiferroic and magnetoelectric metal-organic framework, Adv. Mater. 25, 2284 (2013).

[14] K. Yamauchi, Theoretical prediction of multiferroicity in $\mathrm{SmBaMn}_{2} \mathrm{O}_{6}$, J. Phys. Soc. Japan 82, 043702 (2013).

[15] D. Khomskii, Classifying multiferroics: Mechanisms and effects, Physics 2, 20 (2009).

[16] G. Zhang, S. Dong, Z. Yan, Y. Guo, Q. Zhang, S. Yunoki, E. Dagotto, and J.-M. Liu, Multiferroic properties of $\mathrm{CaMn}_{7} \mathrm{O}_{12}$, Phys. Rev. B 84, 174413 (2011).

[17] R. D. Johnson, L. C. Chapon, D. D. Khalyavin, P. Manuel, P. G Radaelli, and C. Martin, Giant Improper Ferroelectricity in the 
Ferroaxial Magnet $\mathrm{CaMn}_{7} \mathrm{O}_{12}$, Phys. Rev. Lett. 108, 067201 (2012).

[18] N. Terada, Y. S. Glazkova, and A. A. Belik, Differentiation between ferroelectricity and thermally stimulated current in pyrocurrent measurements of multiferroic $\mathrm{MMnO}_{12}(\mathrm{M}=\mathrm{Ca}$, Sr, Cd, Pb), Phys. Rev. B 93, 155127 (2016).

[19] N. J. Perks, R. D. Johnson, C. Martin, L. C. Chapon, and P. G. Radaelli, Magneto-orbital helices as a route to coupling magnetism and ferroelectricity in multiferroic $\mathrm{CaMn}_{7} \mathrm{O}_{12}$, Nat. Commun. 3, 1277 (2012).

[20] See Supplemental Material at http://link.aps.org/supplemental/ 10.1103/PhysRevB.97.144102 for details of sample characterization, single-crystal and Rietveld refinements, and irreducible representation characters.

[21] A. J. Studer, M. E. Hagen, and T. J. Noakes, Wombat: The highintensity powder diffractometer at the OPAL reactor, Phys. B: Condens. Matter 385-386, 1013 (2006).

[22] K. D. Liss, B. Hunter, M. Hagen, T. Noakes, and S. Kennedy, Echidna-The new high-resolution powder diffractometer being built at OPAL, Phys. B: Condens. Matter 385-386, 1010 (2006).

[23] B. J. Campbell, H. T. Stokes, D. E. Tanner, and D. M. Hatch, ISODISPLACE: A web-based tool for exploring structural distortions, J. Appl. Crystallogr. 39, 607 (2006).

[24] B. J. Campbell, J. S. O. Evans, F. Perselli, and H. T. Stokes, Rietveld refinement of structural distortion-mode amplitudes, IUCr Comput. Comm. Newsl. 8, 81 (2007).

[25] J. W. Lewis, J. L. Payne, I. R. Evans, H. T. Stokes, B. J. Campbell, and J. S. O. Evans, An exhaustive symmetry approach to structure determination: Phase transitions in $\mathrm{Bi}_{2} \mathrm{Sn}_{2} \mathrm{O}_{7}, \mathrm{~J}$. Am. Chem. Soc. 138, 8031 (2016).

[26] W. Kabsch, XDS, Acta Crystallogr., Sect. D: Biol. Crystallogr. 66, 125 (2010).

[27] R. Przenioslo, I. Sosnowska, E. Suard, A. Hewat, and A. N. Fitch, Phase coexistence in the charge ordering transition in $\mathrm{CaMn}_{7} \mathrm{O}_{12}$, J. Phys.: Condens. Matter 14, 5747 (2002).

[28] J. P. Attfield, Charge ordering in transition metal oxides, Solid State Sci. 8, 861 (2006).

[29] S. V. Streltsov and D. I. Khomskii, Jahn-Teller distortion and charge, orbital, and magnetic order in $\mathrm{NaMn}_{7} \mathrm{O}_{12}$, Phys. Rev. B 89, 201115 (2014).

[30] P. M. M. Thygesen, C. A. Young, E. O. R. Beake, F. D. Romero, L. D. Connor, T. E. Proffen, A. E. Phillips, M. G. Tucker, M. A.
Hayward, D. A. Keen, and A. L. Goodwin, Local structure study of the orbital order/disorder transition in $\mathrm{LaMnO}_{3}$, Phys. Rev. B 95, 174107 (2017).

[31] T. Tohyama, M. S. Senn, T. Saito, W.-T. Chen, C. C. Tang, J. P. Attfield, and Y. Shimakawa, Valence change of A'-site Mn by A-site doping in $\mathrm{La}_{1-x} \mathrm{Na}_{x} \mathrm{Mn}_{3} \mathrm{Ti}_{4} \mathrm{O}_{12}$, Chem. Mater. 25, 178 (2013).

[32] A. Aimi, D. Mori, K.-I. Hiraki, T. Takahashi, Y. J. Shan, Y. Shirako, J. Zhou, and Y. Inaguma, High-pressure synthesis of Asite ordered double-perovskite $\mathrm{CaMnTi}_{2} \mathrm{O}_{6}$ and ferroelectricity driven by coupling of A-site ordering and the second-order JahnTeller effect, Chem. Mater. 26, 2601 (2014).

[33] S. Zhang, T. Saito, M. Mizumaki, W.-T. Chen, T. Tohyama, and Y. Shimakawa, Site-selective doping effect in $\mathrm{AMn}_{3} \mathrm{~V}_{4} \mathrm{O}_{12}$ $(\mathrm{A}=\mathrm{Na}+\mathrm{Ca} 2+$, and $\mathrm{La} 3+)$, J. Am. Chem. Soc. 135, 6056 (2013).

[34] Y. W. Long, N. Hayashi, T. Saito, M. Azuma, S. Muranaka, and Y. Shimakawa, Temperature-induced A-B intersite charge transfer in an A-site-ordered $\mathrm{LaCu}(3) \mathrm{Fe}(4) \mathrm{O}(12)$ perovskite, Nature (London) 458, 60 (2009).

[35] M. J. Pitcher, P. Mandal, M. S. Dyer, J. Alaria, P. Borisov, H. Niu, J. B. Claridge, and M. J. Rosseinsky, Tilt engineering of spontaneous polarization and magnetization above $300 \mathrm{~K}$ in a bulk layered perovskite, Science 347, 420 (2015).

[36] M. S. Senn, A. Bombardi, C. A. Murray, C. Vecchini, A. Scherillo, X. Luo, and S. W. Cheong, Negative Thermal Expansion in Hybrid Improper Ferroelectric Ruddlesden-Popper Perovskites by Symmetry Trapping, Phys. Rev. Lett. 114, 035701 (2015).

[37] Y. S. Oh, X. Luo, F.-T. Huang, Y. Wang, and S.-W. Cheong, Experimental demonstration of hybrid improper ferroelectricity and the presence of abundant charged walls in $(\mathrm{Ca}, \mathrm{Sr})_{3} \mathrm{Ti}_{2} \mathrm{O}_{7}$ crystals, Nat. Mater. 14, 407 (2015).

[38] K. Yamauchi and P. Barone, Electronic ferroelectricity induced by charge and orbital orderings, J. Phys.: Condens. Matter 26, 103201 (2014).

[39] N. Ikeda, H. Ohsumi, K. Ohwada, K. Ishii, T. Inami, K. Kakurai, Y. Murakami, K. Yoshii, S. Mori, Y. Horibe, and H. Kitô, Ferroelectricity from iron valence ordering in the charge-frustrated system $\mathrm{LuFe}_{2} \mathrm{O}_{4}$, Nature (London) 436, 1136 (2005).

[40] M. S. Senn, J. P. Wright, and J. P. Attfield, Charge order and three-site distortions in the Verwey structure of magnetite, Nature (London) 481, 173 (2012). 